\title{
Spatio-temporal Free-Form Registration of Cardiac MR Image Sequences
}

\author{
Dimitrios Perperidis $^{1}$, Raad Mohiaddin ${ }^{2}$, and Daniel Rueckert ${ }^{1}$ \\ 1 Visual Information Processing Group, Department of Computing, Imperial College London, \\ 180 Queen's Gate, London SW7 2AZ, United Kingdom \\ 2 Royal Brompton and Harefield NHS Trust, Sydney Street, \\ London, United Kingdom
}

\begin{abstract}
In this paper we develop a spatio-temporal registration algorithm for cardiac MR image sequences. The algorithm has the ability to correct any spatial misalignment between the images caused by global differences in the acquisition of the image sequences and by local shape differences. In addition it has the ability to correct temporal misalignment caused by differences in the length of the cardiac cycles and by differences in the dynamic properties of the hearts. The algorithm uses a 4D deformable transformation model which is separated into spatial and temporal components. The registration method was qualitatively evaluated by visual inspection and by measuring the overlap and surface distance of anatomical regions. The results demonstrate that a significant improvement in the alignment of the image sequences is achieved by the use of the deformable transformation model.
\end{abstract}

\section{Introduction}

Cardiovascular diseases are a very important cause of death in the developed world. Their early diagnosis and treatment is crucial in order to reduce mortality and to improve patients' quality of life. Recent advances in the development of non-invasive imaging modalities are enabling the high resolution imaging of the cardiovascular system. Among these modalities, magnetic imaging (MR) is playing an increasingly important role. MR imaging allows not only the acquisition of 3D images which describe the cardiac anatomy but also the acquisition of 4D cardiac image sequences which describe the cardiac anatomy as well as function.

The recent advantages in the development of cardiac imaging modalities have led to an increased need for cardiac registration methods (for recent reviews of cardiac image registration methods see [1] and [2] for a general review of image registration methods). In general, cardiac image registration is a very complex problem due to the complicated non-rigid motion of the heart and the thorax as well as the low resolution with which cardiac images are usually acquired. In the recent years cardiac image registration has emerged as an important tool for a large number of applications. It has a fundamental role in the construction of anatomical atlases of the heart [34]. It has also been used for the analysis of the myocardial motion [5] and for the segmentation of cardiac images [6]. Image registration has been also used for the fusion of information from a number of different modalities such as CT, MR, PET, and SPECT [78]. In addition, cardiac 
image registration is crucial for the comparison of images of the same subject, e.g. before and after pharmacological treatment or surgical intervention. Furthermore, intersubject alignment of cardiac image sequences to the same coordinate space (anatomical reference) enables direct comparisons between the cardiac anatomy and function of different subjects to be made.

While a large number of registration techniques exist for cardiac images, most of these techniques focus on 3D images ignoring any temporal misalignment between the two image sequences. In this paper we extend a 4D cardiac MR image registration method based on voxel similarity measures which has been recently presented [910, 3]. This method will not only bring a number of sequences of cardiac images acquired from different subjects or the same subject (for example short and long axis cardiac image sequences) into the same spatial coordinate frame but also into the same temporal coordinate frame. This allows direct comparison between both the cardiac anatomy of different subjects and the cardiac function to be made. The aim of this contribution is to improve the accuracy of the cardiac MR image sequence registration algorithm by using a spatio-temporal free-form deformation model based on B-Splines. The registration method which has been previously presented [10] had the ability to correct spatial misalignment caused by both global and local differences of the shape of the heart as well as temporal misalignment due to differences in the temporal acquisition parameters but not temporal misalignment caused by different cardiac dynamics. This contribution will enable the correction of temporal misalignment not only caused by differences in the temporal acquisition parameters but also in the cardiac dynamics providing a spatiotemporal deformable registration method for cardiac MR image sequences.

\section{Spatio-temporal Registration}

Since the heart is undergoing a spatially and temporally varying degree motion during the cardiac cycle, 4D cardiac image registration algorithms are required when registering two cardiac MR image sequences. Spatial alignment of corresponding frames of the image sequences (e.g. the second frame of one image sequence with the second frame of the other) is not enough since these frames may not correspond to the same position in the cardiac cycle of the hearts. This is due to differences in the acquisition parameters (initial offset in the acquisition of the first frame and different frequency in the acquisition of consecutively frames), differences in the length of cardiac cycles (e.g. one cardiac cycle maybe longer than the other) and differences in the dynamic properties of the hearts (e.g. one heart may have longer contraction phase and shorter relaxation phase). Spatiotemporal alignment will enable comparison between corresponding anatomical positions and corresponding positions in the cardiac cycle of the hearts. It will also resolve spatial ambiguities which occur when there is not sufficient common appearance in the two 3D MR cardiac images. Furthermore, it can also improve the results of the registration because it is not restricted only to the alignment of existing frames but it can also use sub-frame information.

A 4D cardiac image sequence can be represented as sequence of $n 3 \mathrm{D}$ images $I_{k}(x, y, z)$ with a fixed field of view $\Omega_{I}$ and an acquisition time $t_{k}$ with $t_{k}<t_{k+1}$, in the temporal direction. The resulting image sequence can be viewed as $4 \mathrm{D}$ image 
$I(x, y, z, t)$ defined on the spatio-temporal domain $\Omega_{I} \times\left[t_{1}, t_{n}\right]$. The goal of 4D image registration described in this paper is to relate each point of one image sequence to its corresponding point of the reference image sequence. In this case the transformation $\mathbf{T}:(x, y, z, t) \rightarrow\left(x^{\prime}, y^{\prime}, z^{\prime}, t^{\prime}\right)$ maps any point of one image sequence $I(x, y, z, t)$ into its corresponding point in the reference image sequence $I\left(x^{\prime}, y^{\prime}, z^{\prime}, t^{\prime}\right)$. The mapping used in this paper is of the following form:

$$
\mathbf{T}(x, y, z, t)=\left(x^{\prime}(x, y, z), y^{\prime}(x, y, z), z^{\prime}(x, y, z), t^{\prime}(t)\right)
$$

and can be of a subvoxel displacement in the spatial domain and of a sub-frame displacement in the temporal domain. The 4D mapping can be resolved into decoupled spatial and temporal components $\mathbf{T}_{\text {spatial }}$ and $\mathbf{T}_{\text {temporal }}$ respectively where

$$
\mathbf{T}_{\text {spatial }}(x, y, z)=\left(x^{\prime}(x, y, z), y^{\prime}(x, y, z), z^{\prime}(x, y, z)\right), \mathbf{T}_{\text {temporal }}(t)=t^{\prime}(t)
$$

each of which we choose to be one-to-one mappings. One consequence of this decoupling is that each temporal frame $t$ in image sequence $I$ will map to another temporal frame $t^{\prime}$ in image sequence $I^{\prime}$, ensuring causality and preventing different regions in a 3D image $I_{t}(x, y, z)$ from being warped differently in the temporal direction by $\mathbf{T}_{\text {temporal }}$.

\subsection{Spatial Alignment}

The aim of the spatial part of the transformation is to relate each spatial point of an image to a point of the reference image, i.e. $\mathbf{T}_{\text {spatial }}:(x, y, z) \rightarrow\left(x^{\prime}, y^{\prime}, z^{\prime}\right)$ maps any point $(x, y, z)$ of a particular time frame $t$ in one image sequence into its corresponding point $\left(x^{\prime}, y^{\prime}, z^{\prime}\right)$ of another particular time frame $t^{\prime}$ of the reference image sequence. The transformation $\mathbf{T}_{\text {spatial }}$ consists of a global transformation and a local transformation:

$$
\mathbf{T}_{\text {spatial }}(x, y, z)=\mathbf{T}_{\text {spatial }}^{\text {global }}(x, y, z)+\mathbf{T}_{\text {spatial }}^{\text {local }}(x, y, z)
$$

The global transformation addresses differences in the size, orientation and alignment of the hearts while the local part addresses differences in the shape of the hearts. An affine transformation with 12 degrees of freedom utilizing scaling and shearing in addition to translation and rotation is used as $\mathbf{T}_{\text {spatial }}^{\text {global }}$.

A free-form deformation (FFD) model based on B-splines is used in order to describe the differences in the local shape of the hearts. To define a spline-based FFD we denote the spatial domain of the image volume as $\Omega_{I}=\{(x, y, z) \mid 0 \leq x<X, 0 \leq y<$ $Y, 0 \leq z<Z\}$. Let $\Phi$ denote a $n_{x} \times n_{y} \times n_{z}$ mesh of control points $\phi_{i, j, k}$ with uniform spacing $\delta$. Then, the FFD can be written as the 3D tensor product of the familiar 1D cubic B-splines [11]:

$$
\mathbf{T}_{\text {spatial }}^{\text {local }}(x, y, z)=\sum_{l=0}^{3} \sum_{m=0}^{3} \sum_{n=0}^{3} B_{l}(u) B_{m}(v) B_{n}(w) \phi_{i+l, j+m, k+n}
$$

where $i=\left\lfloor\frac{x}{n_{x}}\right\rfloor-1, j=\left\lfloor\frac{y}{n_{y}}\right\rfloor-1, k=\left\lfloor\frac{z}{n_{z}}\right\rfloor-1, u=\frac{x}{n_{x}}-\left\lfloor\frac{x}{n_{x}}\right\rfloor, v=\frac{y}{n_{y}}-\left\lfloor\frac{y}{n_{y}}\right\rfloor, w=$ $\frac{z}{n_{z}}-\left\lfloor\frac{z}{n_{z}}\right\rfloor$ and where $B_{l}$ represents the $l$-th basis function of the B-spline.One advantage 
of B-Splines is that they are locally controlled which makes them computationally efficient even for a large number of control points. In particular, the basis functions of cubic B-Splines have a limited support, i.e. changing a control point affects the transformation only in the local neighborhood of that control point. This combined spatial deformation model has been introduced by Rueckert et al. [11]. It has been used by a large number of approaches in cardiac MR imaging including for the analysis of the myocardial motion [5], and for the segmentation of the myocardium and the ventricles of cardiac MR image sequences [6].

\subsection{Temporal Alignment}

The temporal part of the transformation consists of a temporal global part, $\mathbf{T}_{\text {temporal }}^{\text {global }}$, and a temporal local part, $\mathbf{T}_{\text {temporal }}^{\text {local }}$ :

$$
\mathbf{T}_{\text {temporal }}(t)=\mathbf{T}_{\text {temporal }}^{\text {global }}\left(t^{\prime}\right)+\mathbf{T}_{\text {temporal }}^{\text {local }}\left(t^{\prime}\right)
$$

$\mathbf{T}_{\text {temporal }}^{\text {global }}$ is an affine transformation which corrects for differences in the length of the cardiac cycles and differences in the acquisition parameters. $\mathbf{T}_{\text {temporal }}^{\text {local }}$ is modeled by a free-form deformation using a 1D B-spline and corrects for temporal misalignment caused by different cardiac dynamic properties (difference in the length of contraction and relaxation phases, different motion patterns, etc). To define a spline-based temporal free-form deformation we denote the temporal domain of the image sequence as $\Omega_{t}=$ $\{(t) \mid 0 \leq x<T\}$. Let $\Phi$ denote a set of $n_{t}$ control points $\phi_{t}$ with a temporal spacing $\delta_{t}$. Then, the temporal free-form deformation can be defined as a 1D cubic B-spline:

$$
\mathbf{T}_{\text {temporal }}^{\text {local }}(t)=\sum_{l=0}^{3} B_{l}(u) \phi_{t_{i+l}}
$$

where $i=\left\lfloor\frac{t}{n_{t}}\right\rfloor-1, u=\frac{t}{n_{t}}-\left\lfloor\frac{t}{n_{t}}\right\rfloor$ and $B_{l}$ represents the 1-th basis function of the B-spline.

$\mathbf{T}_{\text {temporal }}^{\text {local }}$ deforms the temporal characteristics of each image sequence in order to follow the same motion pattern with the reference image sequence. The combined 4D transformation model (equation 1) is the spatio-temporal free-form deformation (STFFD).

\subsection{Voxel-Based Similarity of Image Sequences}

The optimal transformation is found by maximising a voxel based similarity measure, the Normalised Mutual Information (NMI) [12] which is a very commonly used similarity measure in the field of medical image registration. The normalised mutual information of the two image sequences can be calculated directly from the joint intensity histogram of the two sequences over the spatio-temporal domain of overlap $\Omega_{I_{A}} \times\left[t_{A 1}, t_{A n}\right] \cap \mathbf{T}\left(\Omega_{I_{B}} \times\left[t_{B 1}, t_{B n}\right]\right)$. During the optimisation new voxel values are generated in the temporal domain using linear interpolation and trilinear interpolation in the spatial domain. In the first part of the optimization procedure NMI is optimized 
as a function of $\mathbf{T}_{\text {spatial }}^{\text {global }}$ and $\mathbf{T}_{\text {temporal }}^{\text {global }}$ using an iterative downhill descent algorithm. In the second part, NMI is optimized as a function of $\mathbf{T}_{\text {spatial }}^{\text {local }}$ and $\mathbf{T}_{\text {temporal }}^{\text {local }} \mathbf{u s i n g}$ a simple iterative gradient descent method.

\section{Results and Discussion}

To evaluate the spatio-temporal deformable registration algorithm we have acquired fifteen cardiac MR image sequences from healthy volunteers. All image sequences used for our experiments were acquired on a Siemens Sonata 1.5 T scanner using TrueFisp pulse sequence. For the reference subject 32 different time frames were acquired (cardiac cycle of length $950 \mathrm{msec}$ ). Each 3D image of the sequence had a resolution of $256 \times$ $192 \times 46$ with a pixel size of $0.97 \mathrm{~mm} \times 0.97 \mathrm{~mm}$ and a slice thickness of $3 \mathrm{~mm}$. Fourteen $4 \mathrm{D}$ cardiac MR images were registered to the reference subject. The length of the cardiac cycle of these images sequences varied from $300 \mathrm{msec}$ to $800 \mathrm{msec}$. An initial estimate of the global spatial transformation was provided due to the large variety in the position and orientation of the hearts. Since all the image sequences contained almost entire cardiac cycles, the global temporal transformation was calculated in order to compensate the differences in length of the cardiac cycles of the subjects (by matching the first and the last frames of the image sequences). The spacing of the control points of the local transformation were $10 \mathrm{~mm}$ in the spatial domain and $90 \mathrm{msec}$ in the temporal domain.

Figure 1 (a) shows the volume curves of the left ventricle after the optimization of the spatio-temporal global transformation, while 1(b) shows the same volume curves and after the optimization of the spatio-temporal local transformation. The volume of the left ventricles were calculated using segmented images. The images were segmented using an EM-algorithm developed by Lorenzo-Valdés et al. [13]. We can clearly see that with the introduction of the deformable components the hearts are significantly better aligned in the temporal domain. All the hearts in 1)(b) follow a similar motion pattern.

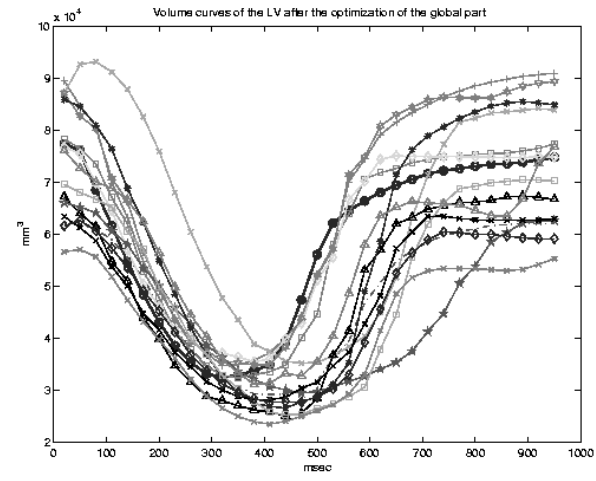

(a)

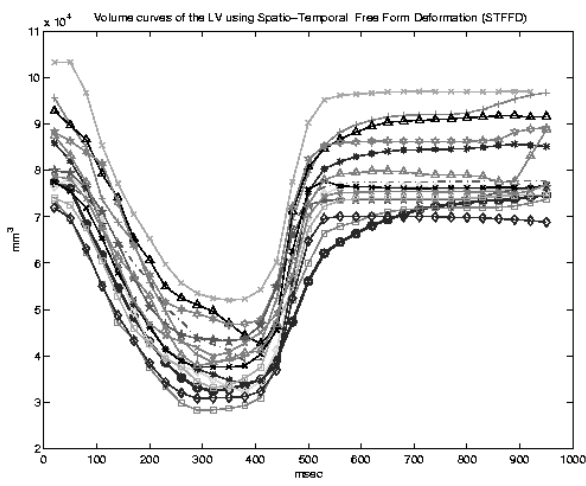

(b)

Fig. 1. The volume curves of the left ventricle for all subjects after optimisation of the global spatio spatio-temporal transformation (a) and the local spatio-temporal registration (b). 


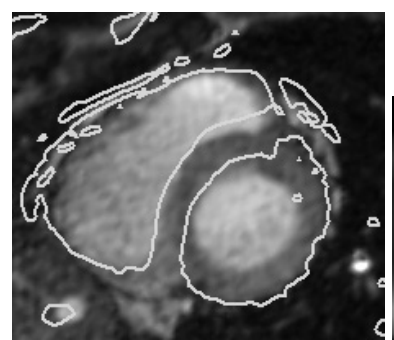

(a)

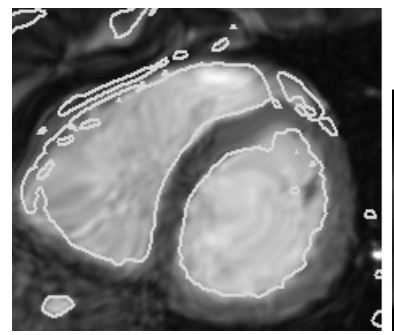

(d)

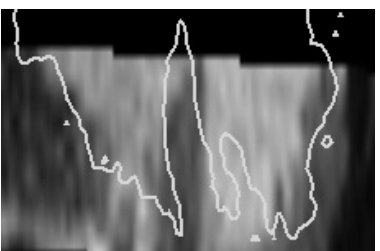

(b)

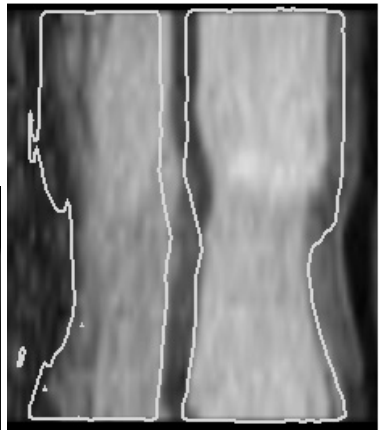

(c)

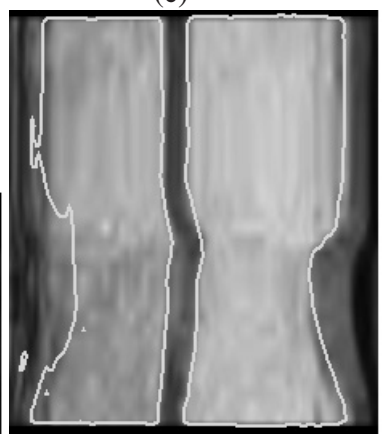

(f)

Fig. 2. Results of the 4D cardiac MR registration algorithm. (a-c) shows the short axis, the long axis and the temporal views after the affine alignment, (d-f) shows the corresponding short axis, long axis and temporal views after the spatio-temporal free-form registration. Animations of the registrations can be found at http://www.doc.ic.ac.uk/ dp1/Conferences/MICCAI04/

Figure 2 provides an example of the spatio-temporal free-form registration. The images in the top row (a-c) are the short axis, the long axis and the temporal views of a frame in the middle of the image sequence after the optimization of the global transformations (affine spatio-temporal registration). The lines in the images represent the contours of the reference image sequence. The images in the bottom row of figure 2 are the same images after spatio-temporal free-form registration. We can clearly see with the introduction of the deformable temporal and spatial transformation there is a significant improvement in the alignment of the image sequences both in the spatial and in the temporal domain. This enables comparisons between corresponding positions in the cardiac cycle to be performed. The blank areas in the long axis views are caused due to a smaller off-plane field of view in the current subject. The quality of the registration in the spatial domain was measured by calculating the volume overlap for the left and right ventricles as well as for the myocardium. The volume overlap for an object $O$ is defined as:

$$
\Delta(T, S)=\frac{2 \times|T \bigcap S|}{|T|+|S|} \times 100 \%
$$


Table 1. The mean volume overlap and surface distance after the affine 4D registration,after the deformable $3 \mathrm{D}$ and after spatio-temporal deformable registration.

\begin{tabular}{|c|c|c|c|c|c|c|}
\hline & \multicolumn{3}{|c|}{ Volume Overlap } & \multicolumn{3}{|c|}{ Surface Distance in $\mathrm{mm}$} \\
\hline Anatomical region & $\begin{array}{c}\text { Affine } \\
\text { 4D }\end{array}$ & \begin{tabular}{|c|} 
Deformable \\
3D
\end{tabular} & $\begin{array}{c}\text { Deformable } \\
\text { 4D }\end{array}$ & $\begin{array}{c}\text { Affine } \\
\text { 4D }\end{array}$ & $\begin{array}{c}\text { Deformable } \\
\text { 3D }\end{array}$ & $\begin{array}{c}\text { Deformable } \\
4 \mathrm{D}\end{array}$ \\
\hline Left ventricle & $76.15 \%$ & $80.95 \%$ & $85.76 \%$ & 4.16 & 3.56 & 2.96 \\
\hline Right ventricle & $77.39 \%$ & $83.87 \%$ & $84.86 \%$ & 4.95 & 4.27 & 3.60 \\
\hline Myocardium & $70.39 \%$ & $71.64 \%$ & $73.86 \%$ & 4.77 & 3.87 & 4.16 \\
\hline
\end{tabular}

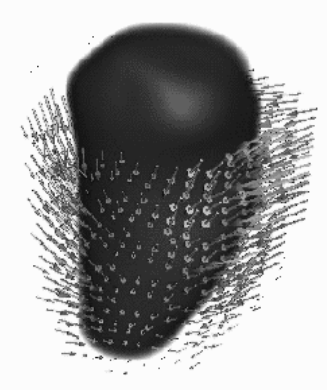

(a)

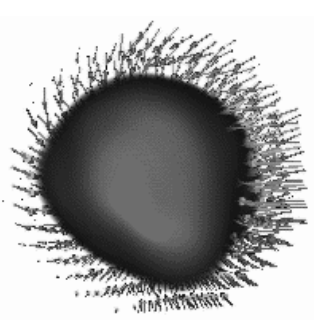

(b)

Fig. 3. Examples of the volume renderings of the probabilistic atlas of the left ventricle with the atlas of the myocardium's deformation [3]. Animations of the atlases can be found at http://www.doc.ic.ac.uk/ dp1/Conferences/MICCAI04/.

Here $T$ denotes the voxels in the reference (target) image part of object $O$ and $S$ denotes the voxels in the other image part of object $O$. We have also calculated the mean surface distance of the above anatomical regions after the affine and the deformable $4 \mathrm{D}$ registration. In order to calculate the overlap of the anatomical structures and the surface distance we used segmented images. Table 1 shows the mean volume overlap and the mean surface distance (in $\mathrm{mm}$ ) for each anatomical region after spatio-temporal affine registration, after $3 \mathrm{D}$ non-rigid registration of the first frames (by matching the first and the last time frames of the image sequences) and after the spatio-temporal deformable registration. The results indicate clearly that the use of the deformable spatial and temporal part provides a significant improvement in the quality of the registration compared to the other two methods.

\section{Conclusions and Further Work}

We have presented an extension to our earlier spatio-temporal registration method [9 10]. In this contribution we extended the registration method by introducing a spatio-temporal deformable transformation model. The proposed registration approach corrects temporal misalignment caused by different acquisition parameters, different length of cardiac cycles and different dynamic properties of the hearts. The approach also corrects spatial 
misalignment caused by global differences in the acquisition of the image sequences and local shape differences. There is a large range of applications for this spatio-temporal registration method. We are planning to use it for building a probabilistic atlas of the cardiac anatomy and function similar to the one we have recently presented [3]. In this case, image registration is used in order to map all the image sequences used for the atlas construction to the same spatio-temporal coordinate system. Then, the atlas can be constructed by averaging the transformed image sequences. Figure 3 provides an example of a 4D probabilistic atlas of the left ventricle with the average deformation of the myocardium [3]. Moreover, the method can be used anytime a comparison between image sequences from the same subject (before and after surgical intervention or pharmacological treatment) or from different subjects (comparison of different cardiac anatomies and functions, atlas based registration) is required. The method was evaluated using fifteen image sequences from healthy volunteers. The results indicate a significant improvement in the temporal and spatial registration of the image sequences with the use of the spatio-temporal deformable transformation model.

\section{References}

1. T. Mäkelä, P. Clarysse, N. Sipila, O.and Pauna, and Q. C. Pham. A review of cardiac image registration methods. IEEE Transcactions on Medical Imaging, 21(9), 2002.

2. B. Zitová and J. Flusser. Image registration methods: a survey. Image and Vision Computing, 21:977-1000, 2003.

3. D. Perperidis, M. Lorenzo-Valdés, R. Chandrashekara, A. Rao, R. Mohiaddin, G.I SanchezOrtiz, and D. Rueckert. Building a 4D atlas of the cardiac anatomy and motion using MR imaging. In 2004 IEEE International Symposium on Biomedical Imaging: From Nano to Macro, 2004.

4. A.F. Frangi, D. Rueckert, J. A. Schnabel, and W.J. Niessen. Automatic construction of multiple-object three-dimensional statistical shape models: Application to cardiac modeling. IEEE Transaction on Medical Imaging, 21(9):1151-1165, 2002.

5. R. Chandrashekara, R. H. Mohiaddin, and D. Rueckert. Analysis of myocardial motion in tagged MR images using nonrigid image registration. In M. Sonka and J. Michael Fitzpatrick, editors, Proceedings of the SPIE International Symposium on Medical Imaging, pages 11681179, San Diego, California USA, 24-28 February 2002.

6. M. Lorenzo-Valdés, G.I. Sanchez-Ortiz, R. Mohiaddin, and D. Rueckert. Atlas based segmentation and tracking of 3D cardiac MR images using non rigid registration. In Fith Int. Conf. on Medical Image Computing and Computer-Assisted Intervention (MICCAI'02), Lecture Notes in Computer Science, pages 642-650. Springer-Verlag, 2002.

7. M.C. Gilardi, G. Rizzo, A. Savi, C. Landoni, V. Bettinardi, C. Rosseti, G. Striano, and F. Fazio. Correlation of SPECT and PET cardiac images by a surface matching registration technique. Computerized Medical Imaging and Graphics, 22(5):391-398, 1998.

8. T. G. Turkington, T. R. DeGradom, M. W. Hanson, and E. R. Coleman. Alignment of dynamic cardiac PET images for correction of motion. IEEE Transaction on Nuclear Science, 44(2):235-242, 1997.

9. D. Perperidis, A. Rao, M. Lorenzo-Valdés, R. Mohiaddin, and D. Rueckert. Spatio-temporal alignment of 4D cardiac MR images. In Lecture Notes in Computer Science: Functional Imaging and Modeling of the heart, FIMH'03, Lyon, France, June 5-6, 2003. Springer.

10. D. Perperidis, A. Rao, R. Mohiaddin, and R. Rueckert. Non-rigid spatio temporal alignment of 4D cardiac MR images. In Lecture Notes in Computer Science: Second International Workshop on Biomedical Image Registration (WBIR). Springer, 2003. 
11. D. Rueckert, L. I. Sonoda, C. Hayes, D.L.G Hill, M.O. Leach, and D.J Hawkes. Nonrigid registration using free-form deformations: Application to breast MR images. IEEE Transactions on Medical Imaging, 18(8):712-721, 1999.

12. C. Studholme, D.L.G. Hill, and D.J. Hawkes. An overlap invariant entropy measure of 3D medical image alignment. Pattern Recognition, 32:71-86, 1999.

13. M. Lorenzo-Valdés, G. I. Sanchez-Ortiz, R. Mohiaddin, and D. Rueckert. Segmentation of 4D cardiac MR images using a probabilistic atlas and the EM algorithm. In Sixth Int. Conf. on Medical Image Computing and Computer-Assisted Intervention (MICCAI'03), Lecture Notes in Computer Science. Springer-Verlag, 2003. 